\title{
SÍNDROME DE CONGESTIÓN PÉLVICA: UTILIDAD DEL TRATAMIENTO LAPAROSCÓPICO
}

Pelvic congestion syndrome: the usefulness of laparoscopic treatment

Hernando Navarro, M.D.*, María Fernanda Escobar, M.D.**, Javier Fonseca, M.D.***

Recibido: agosto 9/2004 - Revisado: septiembre 1/2004 - Aceptado: febrero 7/2005

\section{RESUMEN}

Objetivo: determinar la utilidad de la ligadura de la vena ovárica dilatada por vía laparoscópica en el tratamiento del síndrome de congestión pélvica.

Tipo de estudio: ensayo clínico controlado no aleatorizado.

Institución: Clínica Los Andes, Cali - Colombia.

Pacientes: 70 pacientes con diagnóstico confirmado por laparoscopia de síndrome de congestión pélvica.

Intervenciones: ligadura de la vena ovárica en 28 pacientes (grupo experimental) y manejo médico en 42 pacientes (grupo control).

Resultados: en el grupo experimental se observó una mejoría del dolor del síndrome de congestión pélvica a los 6 meses en 26 de los 28 pacientes versus 11 de 42 pacientes en el grupo control (RR 3,41 IC95\% 2,02-5,76).

Comentarios: las pacientes sometidas a varicocelectomía laparoscópica tienen una mayor probabilidad de curación del dolor en el síndrome de congestión pélvica.

Palabras clave: pelvis, varicocele, dolor pélvico.

\footnotetext{
* Ginecología y Laparoscopia, Profesor Departamento de Ginecología y Obstetricia. Universidad del Valle. Correo electrónico: hnavarro_ newball@hotmail.com. Dirección correspondencia: Cl 9c 50-25, CaliColombia.

** Ginecóloga. Fundación Clínica Valle del Lili.

*** Ginecólogo, Epidemiólogo. Profesor Departamento de Ginecología y Obstetricia. Universidad del Valle.
}

\section{SUMMARY}

Objective: determining the usefulness of ligature of dilated ovarian vein by laparoscope in treating pelvic congestion syndrome.

Type of study: non-randomized controlled clinical trial.

Institution: Clínica Los Andes, Cali, Colombia.

Patients: 70 patients having a diagnosis of pelvic congestion syndrome confirmed by laparoscope.

Interventions: ligature of ovarian vein in 28 patients (experimental group) and medical management in 42 patients (control group).

Results: an improvement in managing pain related to pelvic congestion syndrome was observed in the experimental group after 6 months in 26 out of the 28 patients cf. 11 out of 42 patients in the control group (3.41 RR; 2.02-5.76 95\%CI).

Comments: patients undergoing laparoscopic varicocelectomy had a greater probability of pelvic congestion syndrome becoming cured.

Key words: pelvis, varicocele, pelvic pain.

\section{INTRODUCCIÓN}

El dolor pélvico crónico es una entidad que afecta a cerca de diez millones de mujeres en todo el mundo, y tiene importantes repercusiones sociales y económicas; del total de casos, siete millones permanecen sin diagnóstico etiológico y por lo tanto no reciben un tratamiento adecuado, a pesar de los adelantos tecnológicos disponibles. 
Recientemente se ha reconocido al síndrome de congestión pélvica como responsable del dolor en este grupo de mujeres. ${ }^{1,2}$

El síndrome de congestión pélvica está asociado a la presencia de várices del sistema venoso pélvico y se presenta como dolor opresivo localizado principalmente en uno de los cuadrantes inferiores del abdomen, con sensación de peso permanente que aumenta con las maniobras de valsalba, además de dismenorrea, dispareunia en las primeras 24 horas postcoito, hemorragia uterina anormal e irritabilidad vesical. ${ }^{3-5}$

El diagnóstico clínico se establece mediante historia clínica y examen físico, en donde la presencia de dolor en el punto ovárico, debe orientar a esta entidad. Otros hallazgos incluyen evidencia de congestión cervical con dolor a su movilización, dolor a la compresión bimanual de los anexos y presencia de várices vulvares. La combinación de los signos clínicos y presencia de dolor postcoito tienen una sensibilidad del $96 \%$ y una especificidad del $77 \% .{ }^{4}$

Pese a contar con abundante literatura médica acerca de su manejo, las alternativas terapéuticas efectivas no son muchas; entre las opciones médicas figura la administración de acetato de medroxiprogesterona solo o en combinación con psicoterapia, que se asocia con reducción del dolor pélvico. ${ }^{6}$ También se pueden usar técnicas quirúrgicas como la histerectomía y ooforectomía bilateral, ligadura y resección de la vena ovárica por técnica abierta, embolización de la arteria ovárica y ligadura ovárica por laparoscopia. ${ }^{7-13}$ Respecto a esta última técnica, existen reportes de una serie de casos con buenos resultados, aunque la ausencia de un grupo control no permite obtener conclusiones definitivas. ${ }^{14-16}$

El objetivo de este estudio fue determinar la utilidad de la ligadura de la vena ovárica por laparoscopia en el tratamiento del síndrome de congestión pélvica en la Clínica Los Andes en Cali, Colombia entre noviembre de 1999 a diciembre de 2001.

\section{MATERIALES Y MÉTODOS}

Se realizó un ensayo clínico no aleatorizado (cuasiexperimento) entre mujeres con cuadro clínico de Dolor Pélvico Crónico Tipo Congestivo (DPCC) confirmado con la visualización de várices pélvicas o congestión y aumento de las venas gonadales durante la laparoscopia con la paciente en posición neutra, con baja presión intra abdominal $(<5 \mathrm{mmHg})$. El DPCC fue diagnosticado como la presencia de dolor pélvico de más de 6 meses de evolución que aumentaba con la actividad física, o dismenorrea y dolor postcoital, que mejoraban espontáneamente una vez finalizada la menstruación y con el reposo, respectivamente y adicionalmente generaban incapacidad funcional, laboral, familiar o social por lo menos un día al mes en los últimos 6 meses. Se excluyeron las pacientes con sintomatología de dolor congestivo pero ausencia de várices durante la laparoscopia.

La distribución de las pacientes en el grupo experimental (ligadura de las venas ováricas vía laparoscópica) o en el grupo control (no realización de ligadura de las venas ováricas) se determinó de acuerdo con el tipo de procedimiento empleado por parte del medico tratante. En el grupo experimental los médicos entrenados en la varicocelectomía aplicaron este procedimiento ante la presencia laparoscópica de congestión venosa pélvica, y se asignó como grupo control a las pacientes a quienes durante el mismo período se les realizó laparoscopia por el grupo de médicos que no hizo una intervención quirúrgica específica al varicocele pélvico. Las pacientes firmaron el consentimiento informado de la intervención propuesta por su médico antes de la cirugía.

En el grupo de ligadura de las venas ováricas, se utilizó un laparoscopio operatorio de 10 grados conectado a un láser de $\mathrm{CO}_{2}$ y a un sistema de video. Se usaron 3 ó 4 puertos de entrada: uno de $11 \mathrm{~mm}$ infraumbilical, uno suprapúbico en línea media de $10 \mathrm{~mm}$ y 1 ó 2 laterales de $5 \mathrm{~mm}$. Después de confirmar por laparoscopia el diagnóstico de várices pélvicas con la paciente en posición neutra a baja presión intrabdominal, se la coloca en posición de Trendelemburg, se incide el peritoneo en el lado derecho de la paciente, con láser de $\mathrm{CO}_{2}$ en superpulso a nivel de la pared lateral de la pelvis, 
sobre el músculo psoas, a la altura de la espina iliaca anterior superior, con una adecuada identificación del trayecto del uréter y de la arteria iliaca común. Posteriormente, se levanta el ligamento infundíbulo pélvico para individualizar el paquete vascular: con una pinza atraumática se divulsiona de la arteria el componente venoso y se liga con dos grapas quirúrgicas de titanio a nivel proximal y distal en el trayecto del vaso ovárico. Confirmada la hemostasia, se repite el procedimiento en el lado izquierdo de la paciente, previa movilización del colon sigmoides. En este grupo ante los hallazgos de otro tipo de patología pélvica asociada se realizó el tratamiento específico para dicha entidad. En ninguna paciente se recomendó tratamiento médico para el dolor pélvico.

En las pacientes del grupo control, se realizó tratamiento quirúrgico en caso de otras patologías adicionales al varicocele pélvico. Adicionalmente en todas se recomendó manejo médico con antiinflamatorios no esteroides, venostáticos y recomendaciones como empleo de medias de gradiente de presión ascendente, ejercicio y disminución de peso.

Todas las pacientes fueron evaluadas 6 meses después de la intervención por un observador, quien desconocía el tipo de tratamiento realizado. En esta evaluación se determinó el dolor mediante la escala visual análoga cromática, que representa la intensidad de dolor en una escala de colores, desde el blanco hasta el rojo oscuro. La escala tiene un equivalente numérico de 0 a 10 , en la que el 0 corresponde a la ausencia de dolor y el 10 a dolor intolerable (Escala de dolor. Reunión Colombiana de Anestesiólogos. 1992). En la aplicación de esta escala no tuvo ninguna participación directa el observador y representa la evaluación del dolor por parte de la paciente. Se consideró mejoría del síndrome de congestión pélvica si no se presentaba un día o más mensual, de incapacidad funcional por dolor pélvico en su ambiente familiar, social, laboral y los síntomas en la escala de dolor visual eran menores o igual a 4. Se catalogó como persistencia, si después del procedimiento, se presentaba 1 día o más de incapacidad funcional o más de 5 puntos en la escala cromática de dolor.

La recolección de la información se realizó por parte de uno de los investigadores principales en excel. Para el análisis se exportó la información a EPI-INFO 6. Las variables categóricas fueron comparadas mediante la prueba Ji cuadrado para muestras independientes o mediante el riesgo relativo con su respectivo intervalo de confianza. Se presenta el RR crudo y el RR ajustado por la comorbilidad asociada.

El estudio fue presentado y aprobado por el comité de ética de la institución (Clínica de Los Andes).

\section{RESUILTADOS}

Durante el período de estudio se realizaron 817 laparoscopias operatorias con diagnóstico de dolor pélvico crónico, en la Clínica Los Andes, Cali - Colombia. En setenta pacientes se confirmó el diagnóstico de síndrome de congestión pélvica por varicocele pélvico.

Las participantes se distribuyeron en 2 grupos: 28 fueron asignadas al grupo experimental y 42 al grupo control. No se presentaron complicaciones quirúrgicas o posquirúrgicas en ningún caso.

La distribución por edad de cada grupo no mostró diferencias estadísticamente significativas $(p=0,08)$

(Tabla 1).

Tabla1. Distribución de las pacientes según la edad y el tipo de tratamiento recibido. Noviem-

bre de 1996 a diciembre de 1999. Clínica Los Andes, Cali -Colombia.

\begin{tabular}{|c|c|c|}
\hline $\begin{array}{c}\text { Grupo de } \\
\text { edad (años) }\end{array}$ & $\begin{array}{c}\text { Ligadura de } \\
\text { várice }\end{array}$ & $\begin{array}{c}\text { Otro } \\
\text { tratamiento }\end{array}$ \\
\hline $15-24$ & 5 & 8 \\
\hline $25-34$ & 15 & 21 \\
\hline $35-44$ & 8 & 13 \\
\hline
\end{tabular}

En 34 pacientes el hallazgo exclusivo fue la presencia de várices pélvicas. En 36 casos se encontraba 
otra patología asociada; en 13 se diagnosticó endometriosis, en 4 adherencias pélvicas, en 6 enfermedad pélvica inflamatoria y en 13 adenomiomatosis $(\mathrm{p}=0,03)($ Tabla 2$)$.

\begin{tabular}{|c|r|c|c|}
\hline $\begin{array}{c}\text { Tabla 2. Distribución de las pacientes según el } \\
\text { diagnóstico y el tipo de tratamiento recibido }\end{array}$ \\
\hline & $\begin{array}{r}\text { Liga- } \\
\text { dura de } \\
\text { várice }\end{array}$ & $\begin{array}{c}\text { Otro } \\
\text { tratamiento }\end{array}$ & $\begin{array}{c}\text { To- } \\
\text { tal }\end{array}$ \\
\hline Varicocele pélvico & 19 & 15 & 34 \\
\hline $\begin{array}{c}\text { Varicocele pélvico con } \\
\text { otra patología asociada }\end{array}$ & 9 & 27 & 36 \\
\hline Total & 28 & 42 & 70 \\
\hline
\end{tabular}

El control realizado 6 meses después de la intervención evidenció mejor respuesta (curación de los síntomas) en las pacientes del grupo experimental (RR 3,41 IC95\% 2,02 - 5,76), con relación a quienes no se les realizó ligadura de la vena ovárica. Dos pacientes del grupo experimental que persistían sintomáticas fueron llevadas a histerectomía abdominal total con diagnóstico patológico de adenomiosis y en una tercera paciente se consideró que presentaba oclusión parcial de una de las venas ováricas al persistir alteración del flujo venoso al estudio doppler, e identificación posterior por una segunda laparoscopia de deslizamiento de la grapa quirúrgica. De las 42 pacientes que fueron sometidas a otro tratamiento (controles), 11 presentaron curación de los síntomas, de las cuales 4 fueron tratadas posteriormente a la laparoscopia diagnóstica, con antibióticos por enfermedad pélvica inflamatoria, a 5 se les realizó fulguración de focos endometriósicos leves a moderados, y a 2 se les dió tratamiento hormonal con acetato de medroxiprogesterona (150 mg IM mensual) por 6 meses (Tabla 3).

En el grupo de las 34 pacientes con diagnóstico exclusivo de varicolece pélvico, se realizaron 19 ligaduras de las venas ováricas por laparoscopia; de éstas, 18 presentaron remisión completa de los síntomas. Una paciente persistió con dolor pélvico y posteriormente se confirmó que presentaba síndrome de congestión pélvica, con flujo anterógrado

\begin{tabular}{|c|c|c|c|c|}
\hline \multicolumn{4}{|c|}{$\begin{array}{c}\text { Tabla 3. Distribución de las pacientes según } \\
\text { evolución de los síntomas del síndrome de } \\
\text { congestión pélvica a los } 6 \text { meses de la } \\
\text { intervención }\end{array}$} \\
$\begin{array}{c}\text { Cura- } \\
\text { ción }\end{array}$ & $\begin{array}{c}\text { Persisten- } \\
\text { cia }\end{array}$ & RR & IC95\% \\
\hline $\begin{array}{c}\text { Ligadura } \\
\text { de várice }\end{array}$ & 25 & 3 & & 2,02 a \\
\hline $\begin{array}{c}\text { Otro } \\
\text { tratamiento }\end{array}$ & 11 & 31 & $*$ & 5,76 \\
\hline Total & 37 & 34 & & \\
\hline
\end{tabular}

* Grupo de referencia

al estudio doppler por bloqueo parcial de la vena gonadal. En las 15 pacientes restantes no se realizó varicocelectomía, a tres se les dió tratamiento con acetato de medroxiprogesterona a $150 \mathrm{mg}$ IM cada mes por 6 meses, 2 presentaron mejoría clínica. En las 12 pacientes restantes se hizo recomendaciones para el manejo de insuficiencia venosa, como ejercicio, disminución de peso, tipo de ropa a usar y medias de gradiente, ninguna de estas pacientes refirió mejoría (Tabla 4).

Tabla 4. Distribución de las pacientes con diagnóstico exclusivo de síndrome de congestión pélvica según la evolución de los síntomas a los 6 meses después de la intervención quirúrgica

\begin{tabular}{c|c|c|c|c|}
\hline & $\begin{array}{c}\text { Cura- } \\
\text { ción }\end{array}$ & $\begin{array}{c}\text { Persisten- } \\
\text { cia }\end{array}$ & RR & IC95\% \\
\hline $\begin{array}{c}\text { Ligadura } \\
\text { de várice }\end{array}$ & 18 & 1 & & $\begin{array}{c}1,95 \mathrm{a} \\
25,93\end{array}$ \\
\hline $\begin{array}{c}\text { Otro } \\
\text { tratamiento }\end{array}$ & 2 & 13 & $*$ & \\
\hline Total & 20 & 14 & & \\
\hline
\end{tabular}

* Grupo de referencia

En las 36 pacientes con patología mixta se realizaron 9 ligaduras de las venas ováricas. Seis pacientes presentaron adicionalmente endometriosis mínima a leve y congestión pélvica por lo cual se realizó fulguración de los focos de endometriosis con mejoría en todas ellas, en los 3 casos restantes se presentó adenomiosis y congestión pélvica, y se realizó solo ligadura de las venas ováricas reportando mejoría, 
en una paciente. Posteriormente fueron llevadas a cirugía abierta y se les confirmó diagnóstico patológico de adenomiosis. De las 27 pacientes restantes (grupo control), 7 presentaron endometriosis moderada a severa por lo cual se les realizó fulguración exclusiva, 5 reportaron mejoría de los síntomas de congestión. En 4 casos se diagnosticó adherencias pélvicas realizando adhesiolisis, ninguna presentó mejoría clínica. En 6 se diagnosticó enfermedad pélvica inflamatoria crónica y se les ordenó tratamiento antibiótico con Etidoxina (doxiciclina) 100 mg vía oral/día, por 15 días, con mejoría en 4 pacientes. En 10 casos restantes se diagnosticó adenomiomatosis. En 7 pacientes se realizó neurectomía a nivel de útero sacros, y a 3 manejo expectante, sin mejoría clínica en ninguna de ellas (Tabla 5).

Tabla 5. Distribución de las pacientes con diagnóstico de síndrome de congestión pélvica asociado a otra patología según la evolución de los síntomas a los 6 meses después de la intervención quirúrgica

\begin{tabular}{|c|c|c|c|c|}
\hline & $\begin{array}{c}\text { Cura- } \\
\text { ción }\end{array}$ & $\begin{array}{c}\text { Persisten- } \\
\text { cia }\end{array}$ & RR & IC95\% \\
\hline $\begin{array}{c}\text { Ligadura } \\
\text { de várice }\end{array}$ & 7 & 2 & & $1,23 \mathrm{a}$ \\
\hline $\begin{array}{c}\text { Otro } \\
\text { tratamiento }\end{array}$ & 9 & 18 & $*$ & \\
\hline Total & 16 & 20 & & \\
\hline
\end{tabular}

\section{DISCUSIÓN}

El síndrome de congestión pélvica es una causa reconocida en la literatura mundial de dolor pélvico crónico. Aunque continúa siendo poco diagnosticado, las várices pélvicas son un posible factor etiológico para la presencia del dolor pélvico, como consecuencia de la disminución mecánica del flujo sanguíneo, o reflujo ante la dilatación venosa pélvica. ${ }^{1,2}$

La sintomatología del síndrome de congestión pélvica incluye dispareunia profunda, dismenorrea congestiva, dolor post-coito, y mejoría con el reposo y la elevación de los miembros inferiores. En el examen físico se encuentra sensibilidad a la palpación de un punto localizado entre el ombligo y la espina iliaca anterior o punto ovárico. La confirmación del diagnóstico clínico se puede realizar con venografía pélvica selectiva y ecografía transvaginal con doppler color. ${ }^{3}$ Los tratamientos más utilizados son la obliteración de la vena ovárica con sus colaterales mediante cirugía abierta intra o extraperitoneal o mediante la embolización guiada por fluoroscopia. ${ }^{9-16}$ Actualmente la laparoscopia constituye un método adecuado para confirmar el diagnóstico. A pesar de ser un método invasivo como la venografía, tiene la ventaja de ser una potencial alternativa de tratamiento.

En 1995, Mathis realiza el primer reporte de un caso de una paciente en la cual la laparoscopia fue el procedimiento confirmatorio y la técnica para la ligadura ovárica selectiva como tratamiento para el síndrome de congestión pélvica. ${ }^{14}$ En 1998, Gómez describe una técnica laparoscópica similar aplicada, con buenos resultados, en todas las 25 pacientes con dolor pélvico crónico secundario a varicocele pélvico. ${ }^{16}$

En la presente investigación, la intervención quirúrgica en pacientes con diagnóstico de varicocele pélvico sin otra patología asociada, se relacionó con mejoría de los síntomas de dolor pélvico crónico, efecto sostenido durante el período de observación. El tratamiento médico con acetato de medroxiprogesterona, en 2 de 3 pacientes, demostró efectos benéficos durante el período de observación del tratamiento; la resolución de los síntomas se explica gracias al efecto de la inducción de un estado hipoestrogénico sobre los factores mecánicos vasculares, hecho que favorece la reducción del calibre vascular y disminuye el flujo anterógrado. Dentro de los tratamientos médicos propuestos se incluyen el acetato de medroxiprogesterona, danazol y acetato goserelina, este último con efecto más sostenido en el tiempo, por tener un efecto más persistente en la vasculatura. ${ }^{16,17}$

En este estudio encontramos que las pacientes sometidas a ligadura de las venas ováricas vía laparoscópica presentaron una mayor incidencia de curación de la sintomatología reportada antes 
del procedimiento, con relación a las pacientes en quienes no se realizó el procedimiento. Los mejores resultados con ligadura de las venas ováricas obtenidos en el presente estudio están acordes con reportes previos, en estudios sin grupo control. A pesar de las diferencias encontradas entre los dos grupos en cuanto a la patología asociada al varicocele, el beneficio del tratamiento se mantiene al estratificar en los grupos denominados varicocele puro y varicocele más otras patologías, aunque es notorio que el efecto benéfico fue mayor para las mujeres con varicocele puro, respecto a aquellas que presentaron otras patologías como endometriosis, enfermedad pélvica inflamatoria y adenomiomatosis. En la medida que se identifique con mayor precisión aquellas pacientes con síntomas de congestión pélvica causados por flujo anterógrado de las venas gonadales varicosas, el tratamiento laparoscópico será más efectivo. Ante la presencia de una patología inflamatoria en pacientes con dolor por congestión pélvica, probablemente el tratamiento exclusivo de la patología será suficiente para la mejoría de los síntomas; se cree que eventualmente la congestión venosa es respuesta fisiológica a un proceso inflamatorio crónico pélvico o estados hiper-estrogénicos transitorios o persistentes, y su curación definitiva depende del tratamiento específico de la patología asociada y control hormonal.

Una de las pacientes en el grupo experimental con persistencia de los síntomas, presentó bloqueo parcial de la vena gonadal, explicado probablemente por el deslizamiento de la grapa quirúrgica al no quedar sus extremos en contacto directo, o por el diámetro venoso que superó el tamaño de la grapa. La falla del bloqueo venoso se puede evitar variando la técnica de oclusión venosa con grapa quirúrgica empleando energía eléctrica. Esta oclusión se logra de forma segura con energía bipolar con control de impedancia sobre el tejido (Ligasure, Valleylap ${ }^{\circledR}$, TYCO Health Care). El control de emisión de energía permite un sellamiento completo y seguro en venas hasta de 1,5 cm ancho, sin generar calor en el tejido vecino ni daño o ruptura de la vena.
Este trabajo constituye el primer avance en la evaluación de la utilidad de la varicocelectomía por laparoscopia, mediante un estudio donde se comparan 2 grupos: uno con intervención y otro sin ella. A pesar de ser un ensayo clínico no aleatorizado, debido a que la selección de las pacientes en cada grupo se realizó de acuerdo a las habilidades quirúrgicas del médico tratante, la aplicación de la escala de dolor en cada paciente permite una evaluación más objetiva de la respuesta a la intervención, controlando el posible sesgo del observador.

El síndrome de congestión pélvica se debe enfocar como una entidad clínica distinta, con implicaciones psicológicas y sexuales propias causadas por las expectativas y el desconocimiento de la causa de dolor por parte de la paciente. La generación de un estado hipoestrogénico es la modalidad de tratamiento médico más efectivo. Ante los resultados de este estudio controlado, con 70 pacientes con diagnóstico clínico confirmado por laparoscopia de síndrome de congestión pélvica, la ligadura de las venas ováricas por laparoscopia con remisión total de los síntomas a los seis meses, se constituye en una excelente alternativa de manejo duradero.

\section{REFERENCIAS}

1. Coutier A, Noone A, Geldacre M. General practitioners' referrals to specialist outpatient clinics. I. Why general practitioners refer patients to specialist outpatient clinics. BMJ 1989;299:304-6.

2. Mathias SD, Kuppermann M, Liberman RF, Lipschutz RC, Steege JF. Chronic pelvic pain: prevalence, healthrelated quality $\mathrm{f}$ live and economic correlates. Obstet Gynecol 1996;87:321-7.

3. Beard RW, Highmann J, Pearce J, Reginald PW. Diagnosis of pelvic varicosities in woman with chronic pelvic pain. Lancet 1984;2:946-9.

4. Beard RW, Reginald PW, Wadsworth J. Clinical features of women with chronic lower abdominal pain due to pelvic congestion. Br J Obstet Gynaecol 1988;95:153-61.

5. Perry CP. Current concepts of pelvic congestion and chronic pelvic pain. JSLS 2001;5:105-10.

6. Soysal ME, Soysal S, Vicdan K, Ozar S. A randomized controlled trial of goserelin and medroxyprogesterone 
acetate in the treatment of pelvic congestion. Hum Reprod 2001;16:931-9.

7. Beard RW, Kennedy RG, Gangar KF, Stones RW, Reginal PW, et al. Bilateral oophorectomy and hysterectomy in the treatment of intractable pelvic pain associated with pelvic congestion. Br J Obstet Gynecol 1991;98:988-92.

8. Edlundh KO. Pelvic varicosities in women: a preliminary report. Acta Obstet Gynecol Scand 1964;43:399-407.

9. Rundqvist E, Sandholm LE, Larsson G. Treatment of pelvic varicosities causing lower abdominal pain with extraperitoneal resection of the left ovarian vein. Ann Chir Gynaecol 1984;1:339-41.

10. Venbrux AC, Lambert DL. Embolization of the ovarian veins as a treatment with chronic pelvic pain caused by pelvic venous incompetence (pelvic congestion syndrome). Curr Opin Obstet Gynecol 1999;11:395-9.

11. Maleux G, Stockx L, Wilms G, Marchal G. Ovarian vein embolization for the treatment of pelvic congestion syndrome: long-term technical and clinical results. J Vasc Interv Radiol 2000;11:859-64.
12. Venbrux AC, Chang AH, Kim HS, Montague BJ, Hebert JB, Arepally A, et al. pelvic congestion syndrome (pelvic venous incompetence): impact of ovarian and internal iliac vein embolotherapy on menstrual cycle and chronic pelvic pain. J Vasc Interv Radiol 2002;13:171-8.

13. Scultetus AH, Villavicencio JL, Gillespie DL, Kao TC, Richman NM. The pelvic venous syndromes: analysis of our experience with 57 patients. J Vasc Surg 2002;36:881-8.

14. Mathis BV, Miller JS, Lukens ML, Paluzzi MW. Pelvic congestion syndrome: a new approach to unusual problem. Am Surg 1995;61:1016-8.

15. Grabham JA, Barrie WW. Laparoscopic approach to pelvic congestion syndrome. Br j Surg 1997;89:1264.

16. Gómez G. Varicocelectomia por laparoscopia: descripción de una nueva técnica y su acción sobre el dolor pélvico. Med Reprod 1998;1:14-7.

17. Navarro H. Dolor pélvico crónico. En: Cifuentes R. Ginecología y obstetricia basadas en la evidencias. Bogotá: Editorial Distribuna; 2002. p. 659-70. 